\title{
Multi-Vision Animation Scene Design Based on VR Technology
}

\author{
Huawen Zhao a,*, Limin Guo, Yanhua Liu \\ Jiangxi College of Applied Technology, Ganzhou, Jiangxi, 341000, China \\ a53998275@qq.com \\ *corresponding author
}

Keywords: VR Technology, Multi Vision, Animation, Scene Design

\begin{abstract}
Some difficult problems will be encountered in the study of complex real scene, and the method of multi-vision animation image reconstruction can be effectively solved. Using the method of $3 \mathrm{D}$ reconstruction, the virtual animation image is established, the feature points are extracted in the animation image of each viewpoint, and after matching, the 3D animation image reconstruction can be completed. If the traditional method is adopted, it cannot only obtain the 3D point set of each frame of the image, but also adopt the fusion processing method, but the ideal reconstruction effect cannot be obtained if the feature point is not corrected in the concrete work. This thesis focuses on the design of multi-vision animation scene based on VR technology.
\end{abstract}

\section{Introduction}

The development of computer graphics and image technology is accelerating, the scope of application is expanding, in addition to the industrial and agricultural fields, the defense and military fields have also increased the utilization of this technology, so this technology has attracted much attention. The so-called 3D scene technology is a simulation technology, its accuracy is very high, mainly used in the design of dynamic animation scene. In the production of 3D simulation scene, it is not only flexible, but also has high accuracy, and the tracking effect is good[1]. The tracking effect obtained by using multi-vision tracking technology is not only true, but also accurate and highly concerned. However, because the complexity of the real scene of dynamic animation is very high, it is challenging to make realistic animation images. This requires building a 3D model of an animated image.

\section{3D Reconstruction of Visual Animated Images Commonly Used at Present}

Currently commonly used 3D reconstruction of visual animation The method is good The tracking effect, which is based on Affine invariant Based on the reconstruction method, according to Affine invariant theory Proceeding detection, combined use Sparse animation scene Constitute, in the animation scene in the plane area to make Dense diffusion matching To do so, we must adopt Geometric constraints Method, combined with use Corresponding point approximation constraint based on Match The results 3D reconstruction of multi-vision animation Work done.There's something visual about this approach True, but there are shortcomings, that is Calculation complex, it takes a long time.If Rank transform is used in the reconstruction of 3D animation Technology, the rest Weighted Average Theory Combined, will Grayscale animated images Rank transform As Match the primitive, you can get A dense view of an animated image, followed by an image Spatial coordinates Do it, make it Scene 3D Point Cloud Map It is possible to generate three-dimensional graphs Coordinates Make it, rebuild it Multi-vision animated images Mission accomplished.The 3D animation reconstructed by this method is highly Accuracy, but there is also a problem Low efficiency. What if Depth confidence estimation theory will be Multi-vision animated images 3D Re-established, you can use Non-uniform sampling Way will be 3D point set per frame extracted, in Depth confidence estimation theory will be Three - dimensional point set fusion 3D point cloud of animated images acquisition, animation 3D geometric model You can get it.The advantage of this 
approach is that it will Between multi-frame images Existence Redundant 3D points Exclude, but in the process of reconstruction of the scene is very demanding, will be subject to the scene Limitations This work is not perfect[2].

\section{Sensory Effects of Multi-Vision Animation Based on VR}

\subsection{Multi-Vision Animation for Vr Technology Good Sensory Effect}

Interactive animation Using VR technology in design can make animation Color, light Constituted Graphics For the audience is more attractive, animated characters can also be the emotional foil.VR based technology animation Can produce good interaction effect, can bring to audience Visual perception Force, also available Touch On the sensory experience, smell On the sensory experience, taste On the sensory experience.Tom and Jerry, for example, a classic cartoon Tom ate the chili After that, face Turn red gradually, audience When I see this, I feel it On the palate It's spicy.This animation experience through Visual language Reaction to the taste, formed Interaction The effect of making animation People image of More plump, and Plot More intension, the audience's viewing experience is also enhanced.

\subsection{Multi-Vision Animation for Vr Technologies Can be Achieved Multi-Sensory Connectivity}

From the current situation of animation design based on VR technology, due to the limitations of technology, the production process of animation needs to consider more about whether the narrative is complete and whether the content is full, and the interaction effect is not reflected. Animation language is the result of visual interaction, animation creators and audiences use this language to communicate, to obtain effective communication, we need to use VR technology reasonably. The theme information conveyed by animation works is also realized by this means. The application of VR technology can make the visual language conveyed by animation easier to understand and understand, and the design is used effectively, which makes the animation more expressive. The application of VR technology in animation creation can establish the connection between the visual and sensory of animation.For example, the smartphone animation, the image can be zoomed in as needed, people in the operation, just use the finger drag. Based on the interactive effect formed by VR technology, multi-vision animation scene design is needed.

\section{Matching Method for 3D Reconstruction of Visual Animation Images}

\subsection{Extracting Feature Points From Animated Images and Match Method}

Rebuilding Multi-vision animated images Do well when you are Calibration Work, these need to be Calibration theory Fully utilize calibration Multi-vision animated images obtained Calibration module Internal parameters and External parameter, Harris corner detection operator Make the most of it, extract each Viewpoint Animation The feature point of the Minimum squared median method Then you can Feature points Extract it, then proceed Calibration[3].

\subsection{Obtain Sparse Beam Adjustment Camera Parameters}

When reconstructing a multi-vision animation image, it can be constructed by using threedimensional technology, which can be expanded according to the matching points between a large number of stable animation images. At this time, we need to play the role of sparse beam adjustment theory to obtain the position parameters and attitude parameters, which are the parameters of multi-vision animation sequence images. Reconstructing the multi-vision animation image, each spatial point is projected in the way of multiple images. If the projection matrix of multi-vision animation is irrelevant, the sparse structure can be fully utilized, and the result can be obtained after quick iteration, so that the camera parameters of multi-vision can be obtained, and the coordinate of animation image point can also be obtained. so that the multi-vision animated image can be reconstructed. 


\section{Experimental Results of 3D Reconstruction of Visual Animation Images}

The above contents are verified by experimental method. Mobile camera From multiple angles Sequence of animated images Image resolution for animation Can reach $1200 \mathrm{x} 1600 \mathrm{dpi}$, using MATLAB software It can be described sparse beam adjustment method the results obtained in the application And bilateral filtering obtained from the Results.

With the rational use of these two methods, the 3D reconstruction of multi-vision animation images can be experimented, and the efficiency of reconstruction can be compared. (Figure 1:3D Image Reconstruction)

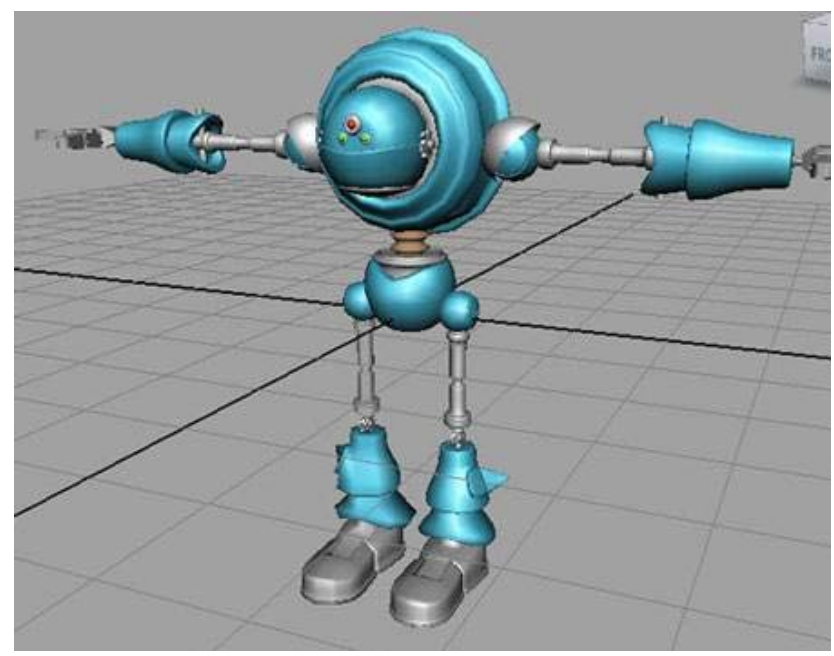

Figure 1 Comparison of 3D reconstruction efficiency of animated images using different methods

Through the analysis of Figure 1, it is clear that sparse beam adjustment method Making the most of it Multi-vision animated images And bilateral filtering There are more obvious advantages in comparison, mainly due to the use of sparse beam adjustment method The image feature points are extracted using Corner detection operator Can be achieved[4]. Based on the sparse beam adjustment theory, the position parameters and attitude parameters of the multi-vision animation image sequence can be obtained. In the application of this method, there is no need for repeated diffusion or filtering, so the efficiency is very high.

Using two methods for image reconstruction, analyzing the number of feature points of the animated image can be extracted, the reconstruction effect of the image can be measured, and the time (ms) of the reconstruction run, the matching logarithm (pair) and the matching rate (\%) of the feature points of the image are compared to describe the effectiveness of the $3 \mathrm{~d}$ reconstruction of the image. See tables 1 and 2.

Table 1 Time comparison table

\begin{tabular}{|c|c|c|}
\hline Group & t/MS & T/MS \\
\hline Group 1 & 556 & 52 \\
\hline Group 2 & 1034 & 101 \\
\hline Group 3 & 1567 & 156 \\
\hline 4 groups & 2012 & 208 \\
\hline Group 5 & 2544 & 255 \\
\hline
\end{tabular}

Table 2 Feature point matching comparison table

\begin{tabular}{|c|c|c|c|c|}
\hline Group & n/Yes & W/\% & N/Yes & S/\% \\
\hline Group 1 & 210 & 25.13 & 421 & 19.23 \\
\hline Group 2 & 241 & 30.52 & 545 & 21.52 \\
\hline Group 3 & 297 & 41.25 & 611 & 25.18 \\
\hline 4 groups & 405 & 52.11 & 735 & 30.52 \\
\hline Group 5 & 209 & 60.52 & 344 & 34.69 \\
\hline
\end{tabular}

Comparison results Look at the description Table 1 and Table 2. 
Where, $\mathrm{t}$ means The running time of the bilateral filtering method is expressed by $\mathrm{T}$ Sparse beam adjustment method running time, $\mathrm{S}$ is expressed as Represents the matching rate of the method, and N means Sparse Beam Adjustment Method Matching Number and Bilateral Filtering Method Matching number In terms of $\mathrm{n}$, the matching rate expressed in $\mathrm{w}$.

By comparison can be clear, use sparse beam adjustment method This is due to the fact that this method will Calibration theory Make the most of it and use the Harris corner detection operator Can be extracted Image of each view animation using the Minimum squared median method The feature points can be extracted Match and Calibration, available Location of an animated image sequence image Parameters and Posture parameters, reconstruction Multi-vision animated images on this basis.

\section{Conclusion}

Through the above research can be clear, for animation image spatial plane matching points extraction, if the traditional method, it is difficult to obtain good results, mainly reflected in the lack of continuity of animation images, visual to give people a intermittent feeling. Building on the theory of sparse beam adjustment to re-establish the multi-vision animation image can be quickly said multi-vision animation image is established, and has a good three-dimensional effect, not only real-time, but also a certain reliability.

\section{References}

[1] Ren, Shan. Explore the influence of VR technology on visual style of 2 D animated films. Tomorrow Fashion, no. 12, pp. 244-246, 2017.

[2] Ning, Ruixin., Zhu, Zunjie., Shao, Biyao. Virtual reality and augmented reality fusion technology based on vision. Science and Technology Bulletin, vol. 36, no. 09, pp. 25-31, 2018.

[3] Zhang, Xiaoming. Application of Visual Composition Language in Animation Multimedia Creation. Art View, no. 01, pp. 138-139, 2017.

[4] Yang, Xiaolan., Qian, Cheng. Research on 3 D Dynamic Laser Imaging Based on VR Technology. Laser Journal, no. 05, pp. 58-59, 2018. 